\title{
Some Remarks on Complex Hamiltonian Systems
}

\author{
R. S. Kaushal ${ }^{\dagger}$ and H. J. Korsch ${ }^{\ddagger *}$ \\ $\dagger$ Dept. of Physics \& Astrophysics, Univ. of Delhi, Delhi - 110007, India \\ $\ddagger$ FB Physik, Univ. Kaiserslautern, D-67653 Kaiserslautern, Germany
}

August 17, 2000

\begin{abstract}
The analyticity property of the one-dimensional complex Hamiltonian system $H(x, p)=H_{1}\left(x_{1}, x_{2}, p_{1}, p_{2}\right)+i H_{2}\left(x_{1}, x_{2}, p_{1}, p_{2}\right)$ with $p=p_{1}+i x_{2}, x=x_{1}+i p_{2}$ is exploited to obtain a new class of the corresponding two-dimensional integrable Hamiltonian systems where $H_{1}$ acts as a new Hamiltonian and $H_{2}$ is a second integral of motion. Also a possible connection between $H_{1}$ and $H_{2}$ is sought in terms of an auto-Bäcklund transformation.
\end{abstract}

PACS: 02.90.+p; 03.20.+i

Keywords: Complex Hamiltonian systems; Integrable systems; $\mathcal{P} \mathcal{T}$-symmetry

${ }^{*}$ Corresponding author; e-mail address: korsch@physik.uni-kl.de 
With a view to having a better theoretical understanding of several newly discovered [1, 2] phenomena there has been [2]-[10] considerable interest in recent years in the study of complex Hamiltonian systems in one space dimension described by the Hamiltonian $H(x, p)$. For this purpose, several methods of complexification are used. One type of complexification which has been known [9] for a long time in the literature and now discussed in several textbooks on quantum mechanics is in the form $z=p+i \omega x, z *=p-i \omega x$, which is in particular well suited for the oscillator problem, as well as its generalized version $[3,4]$ by introducing two complex variables $u=x / b+i p / c$ and $v=x / b-i p / c$, where $b$ and $c$ are complex numbers. Note that in all these cases the complexity arises mainly through the parameter space.

Recently, following the work of C. M. Bender and his coworkers [5, 6] one-dimensional complex Hamiltonian systems have been studied rigorously through the combined parity $(\mathcal{P}: x \rightarrow-x, p \rightarrow-p)$ and time reversal $(\mathcal{T}$ : $x \rightarrow x, p \rightarrow-p, i \rightarrow-i$ ) operations. The corresponding quantum Hamiltonian, which now becomes non-hermitian, is found to yield real eigenvalues for a certain parametric domain if the Hamiltonian is $\mathcal{P} \mathcal{T}$-symmetric. Here, the complex Hamiltonian is typically introduced from the beginning, i.e., $H(x, p)$ is complex even for real values of $x$ and $p$. Boundary conditions are formulated in complex $x$-space and hence the extension to a complex classical phase space appears naturally.

In most cases, however, the system under study is originally a real valued classical Hamiltonian $H(x, p)$ function, defined for real $x$ and $p$ or, quantum mechanically, a hermitian operator. An extension to complex (phase) space is sometimes required for particular purposes. In a semiclassical analysis such a treatment can account for classically forbidden processes [11]. In other situations a complexification can be used to describe resonances as complex energy eigenvalues and eigenstates of the Hamiltonian both quantum mechanically [2] by so-called complex scaling methods and semiclassically [12] by Hamiltonian dynamics at complex energies.

In any case, an understanding of the classical dynamics in the complexified phase space is essential also for the quantum dynamics. Here we will confine ourselves to the classical system. Following $[3,4]$ we define $x$ and $p$ as

$$
x=x_{1}+i p_{2}, \quad p=p_{1}+i x_{2} .
$$

Here the real phase plane $(x, p)$ is replaced by a complex space $\left(x_{1}, x_{2}, p_{1}, p_{2}\right)$ with two additional degrees of freedom, namely $x_{2}$ and $p_{2}$. The $\mathcal{P} \mathcal{T}$-symmetry 
operations are

$$
\begin{array}{ll}
\mathcal{P}: & \left(x_{1}, x_{2}, p_{1}, p_{2}\right) \longrightarrow\left(-x_{1},-x_{2},-p_{1},-p_{2}\right) \\
\mathcal{T}: & \left(x_{1}, x_{2}, p_{1}, p_{2}\right) \longrightarrow\left(x_{1}, x_{2},-p_{1},-p_{2}\right), \quad i \longrightarrow-i .
\end{array}
$$

Clearly, the Hamiltonian $H(x, p)$, after using (1), can be expressed as

$$
H(x, p)=H_{1}\left(x_{1}, x_{2}, p_{1}, p_{2}\right)+i H_{2}\left(x_{1}, x_{2}, p_{1}, p_{2}\right) .
$$

Note that for a time independent Hamiltonian $H(x, p)$ is a constant of motion and so are its real and imaginary parts $H_{1}$ and $H_{2}$ separately.

If $H$ is an analytic function of the two complex variables $x$ and $p$, then $H_{1}$ and $H_{2}$ satisfy the two sets of Cauchy-Riemann equations

$$
\begin{array}{ll}
\frac{\partial H_{2}}{\partial p_{2}}=\frac{\partial H_{1}}{\partial x_{1}}, & \frac{\partial H_{2}}{\partial x_{1}}=-\frac{\partial H_{1}}{\partial p_{2}} \\
\frac{\partial H_{2}}{\partial x_{2}}=\frac{\partial H_{1}}{\partial p_{1}}, & \frac{\partial H_{2}}{\partial p_{1}}=-\frac{\partial H_{1}}{\partial x_{2}},
\end{array}
$$

which imply a vanishing Poisson bracket

$$
\left[H_{1}, H_{2}\right]=\frac{\partial H_{1}}{\partial x_{1}} \frac{\partial H_{2}}{\partial p_{1}}-\frac{\partial H_{1}}{\partial p_{1}} \frac{\partial H_{2}}{\partial x_{1}}+\frac{\partial H_{1}}{\partial x_{2}} \frac{\partial H_{2}}{\partial p_{2}}-\frac{\partial H_{1}}{\partial x_{2}} \frac{\partial H_{2}}{\partial p_{2}}=0
$$

i.e. these constants of motion are in involution.

It is interesting to note here that this result can be derived in a different way: The complex equations of motion

$$
\dot{x}=\frac{\partial H}{\partial p}, \quad \dot{p}=-\frac{\partial H}{\partial x}
$$

are transformed into [3]

$$
\begin{aligned}
& \dot{x}_{1}=\frac{\partial H_{1}}{\partial p_{1}}, \quad \dot{p}_{1}=-\frac{\partial H_{1}}{\partial x_{1}} \\
& \dot{x}_{2}=\frac{\partial H_{1}}{\partial p_{2}}, \quad \dot{p}_{2}=-\frac{\partial H_{1}}{\partial x_{2}}
\end{aligned}
$$

i.e., the Hamiltonian equations for a Hamiltonian $H_{1}\left(x_{1}, x_{2}, p_{1}, p_{2}\right)$ with two degrees of freedom $x_{j}$ and canonical momenta $p_{j}$. This motivates the notations $p_{2}, x_{2}$ as introduced in Eq. (1) for the imaginary parts. The equations of motion (7) can be written in the compact form

$$
\dot{y}=J \nabla_{y} H_{1}(y)
$$


with $y=\left(x_{1}, x_{2}, p_{1}, p_{2}\right)$ and the symplectic unit matrix $J=\left(\begin{array}{cc}0 & I \\ -I & 0\end{array}\right)$ ( $I$ is the two-dimensional unit matrix).

The two-dimensional system with Hamiltonian $H_{1}$ is integrable [13], i.e. there exist two independent integrals of motion, which are in involution, namely $H_{1}$ and $H_{2}$. First, we see from the Cauchy-Riemann equations (4) that $\dot{H}_{2}=\left[H_{1}, H_{2}\right]=0$. Moreover, $H_{1}$ and $H_{2}$ are independent, i.e. the two vector fields

$$
v_{j}=J \nabla_{y} H_{j}(y), \quad j=1,2
$$

are linearly independent.

Other conditions which $H_{1}$ and $H_{2}$ have to satisfy - as a by-product of Eq. (4) - are [14]

$$
\begin{aligned}
& \frac{\partial^{2} H_{j}}{\partial x_{1}^{2}}+\frac{\partial^{2} H_{j}}{\partial p_{2}^{2}}=0, \quad \frac{\partial^{2} H_{j}}{\partial x_{1} \partial p_{1}}+\frac{\partial^{2} H_{j}}{\partial x_{2} \partial p_{2}}=0 \\
& \frac{\partial^{2} H_{j}}{\partial x_{2}^{2}}+\frac{\partial^{2} H_{j}}{\partial p_{1}^{2}}=0, \quad \frac{\partial^{2} H_{j}}{\partial x_{1} \partial x_{2}}-\frac{\partial^{2} H_{j}}{\partial p_{1} \partial p_{2}}=0
\end{aligned}
$$

for $j=1,2$, i.e. $H_{1}$ and $H_{2}$ are bi-harmonic functions and solve the Laplace equation $\Delta_{y} H_{j}=0$. As a consequence, they cannot have a minimum in phase space.

After having this much understanding of the complex Hamiltonian $H(x, p)$ the following remarks are in order:

1. It is well known [15] that there has been a scarcity of integrable systems in two dimensions in the sense that a second invariant (beside the Hamiltonian of the system) does not exist for almost all cases. Even if it exists for some, often its construction becomes a difficult task. The above survey concerning the complex Hamiltonian systems in one dimension gives a clue to such a construction, at least for a certain class of exotic two-dimensional Hamiltonian systems. (Exotic in the sense that they have yet to be identified with the Hamiltonian of a two-dimensional physical system).

As a matter of fact, if one identifies the structure of $H_{1}$ with the Hamiltonian of a given two-dimensional system, then in view of (5), one can say that the second invariant for this system is $H_{2}$. In Table 1 we provide a list of such $H_{1}$ and $H_{2}$ corresponding to a given complex Hamiltonian $H$ of Eq. (3) and note that all the cases conform not only to condition (5) but also to conditions (10). Most of the systems also satisfy the $\mathcal{P} \mathcal{T}$-symmetry, except for case 8. However, our present prescription works for all those structures which can be written in the form (3) using (1). 
2. The cases discussed above and listed in Table 1 just offer some sort of a consistency check for the mathematical setting through Eqs. (3) - (10). Now the following question arises: Can we determine $H_{2}$ (or, say, $H_{1}$ ) if $H_{1}$ (or, say, $\mathrm{H}_{2}$ ) is given along with the conditions (4)? The answer is yes. It is not that for every $H_{1}$ such a function $H_{2}$ can be constructed (or vice versa). In fact $H_{1}$ has to satisfy the conditions (10), only then the construction of $\mathrm{H}_{2}$ becomes possible.

It may be reminded that the Cauchy-Riemann conditions (4) along with (10) offer an example of an auto-Bäcklund transformation. In that there exists [16] a definite prescription to determine $H_{2}$ (the integral of motion) from $H_{1}$ (the Hamiltonian). The cases listed in Table 1 all follow this prescription. Here, however, we present some more cases in which $H_{2}$ is constructed from $H_{1}$ using the same prescription. As we have seen from the case 4 (cf. Table 1), the explicit dependence of $H$ (or for that matter of $H_{1}$ and $H_{2}$ ) on $t$ does not disqualify the system for the above mentioned mathematical setting. Therefore, we note that the couplings in the following examples can as well be time dependent.

(i) Consider the two-dimensional Hamiltonian

$$
H_{1}=\frac{1}{2}\left(p_{1}^{2}+p_{2}^{2}\right)-\frac{1}{2}\left(x_{1}^{2}+x_{2}^{2}\right)+\beta\left(p_{1} p_{2}+x_{1} x_{2}\right)+\gamma\left(x_{1} p_{1}-x_{2} p_{2}\right),
$$

which corresponds to a pair of inverted harmonic oscillators with (real) couplings $\beta$ and $\gamma$. One can verify that this $H_{1}$ satisfies (10). The prescription of a Bäcklund transformation can be used to construct $H_{2}$. For this purpose, using $H_{1}$ from (11), Eqs. (4) can be expressed as

$$
\begin{array}{ll}
\frac{\partial H_{2}}{\partial p_{2}}=-x_{1}+\beta x_{2}+\gamma p_{1}, & \frac{\partial H_{2}}{\partial x_{1}}=-\left(p_{2}+\beta p_{1}-\gamma x_{2}\right) \\
\frac{\partial H_{2}}{\partial x_{2}}=+p_{1}+\beta p_{2}+\gamma x_{1}, & \frac{\partial H_{2}}{\partial p_{1}}=-\left(-x_{2}+\beta x_{1}-\gamma p_{2}\right) .
\end{array}
$$

Integration of these equations immediately yields the solution

$$
H_{2}=\left(p_{1} x_{2}-x_{1} p_{2}\right)+\beta\left(x_{2} p_{2}-x_{1} p_{1}\right)+\gamma\left(p_{1} p_{2}+x_{1} x_{2}\right) \text {. }
$$

Note the interchange of couplings in $H_{2}$ compared to that in $H_{1}$. Further, it can be easily seen that this form of $H_{2}$ conforms to Eq. (10). As Eqs. (11) and 
(14) have to represent the real and imaginary parts of a complex Hamiltonian $H(x, p)$, then $H(x, p)$ can be obtained easily in a very simple form as

$$
H \equiv H_{1}+i H_{2}=\frac{1}{2}\left(p^{2}-x^{2}\right)+b^{*} p x
$$

where $b^{*}=\gamma-i \beta$ and the relations (1) along with $x^{*}=x_{1}-i p_{2}, p^{*}=p_{1}-i x_{2}$ are used.

(ii) Now consider the Hamiltonian corresponding to the coupled oscillators of equal mass $m$ and imaginary frequency $i \omega$ satisfying $\omega^{2}=1 / m$, namely

$$
H_{1}=\frac{\omega^{2}}{2}\left(p_{1}^{2}+p_{2}^{2}-x_{1}^{2}-x_{2}^{2}\right)+f\left(p_{1} x_{2}-p_{2} x_{1}\right)
$$

( $f$ real). As before in Example (i), $H_{2}$ can be derived from Eqs. (4) by integration to give

$$
H_{2}=\left(1+\omega^{2}\right)\left(p_{1} x_{2}-p_{2} x_{1}\right)-\frac{f}{2}\left(p_{1}^{2}+p_{2}^{2}-x_{1}^{2}-x_{2}^{2}\right) .
$$

The corresponding complex $H(x, p)$ can be obtained as

$$
H \equiv H_{1}+i H_{2}=\frac{1}{4}\left(p^{2}-p^{* 2}-x^{2}+x^{* 2}\right)-\frac{i}{2}\left(f+i \omega^{2}\right)\left(p^{2}-x^{2}\right) .
$$

The following two examples consist of very simple two-dimensional Hamiltonian structures. Note that the utility of these structures (or their variants) has recently been emphasized by t'Hooft [17] and Blasone et al. [18, 19] in the context of the so-called holographic principle and in the treatment of quantum gravity as a dissipative and deterministic system. Although the following two examples can be considered as the special cases of Example (i), we would like to give here the relevant results to make the subsequent discussion more effective.

(iii) Consider the two-dimensional Hamiltonian

$$
H_{1}=\alpha\left(p_{1} p_{2}+x_{1} x_{2}\right)+\beta\left(p_{2} x_{2}-p_{1} x_{1}\right)
$$

( $\alpha, \beta$ real), for which the $H_{2}$-function can be derived as in Example (i) as

$$
H_{2}=\alpha\left(p_{2} x_{2}-p_{1} x_{1}\right)-\beta\left(p_{1} p_{2}+x_{1} x_{2}\right),
$$


and the corresponding complex Hamiltonian is given by

$$
H \equiv H_{1}+i H_{2}=-b p x
$$

where $b=\beta+i \alpha$ is a complex constant.

(iv) Lastly, consider the simple two-dimensional Hamiltonian (a measure of the angular momentum) used by t' Hooft [17] as

$$
H_{1}=x_{2} p_{1}-x_{1} p_{2}
$$

for which

$$
H_{2}=\frac{1}{2}\left(x_{1}^{2}+x_{2}^{2}-p_{1}^{2}-p_{2}^{2}\right)
$$

can be obtained as before and the corresponding complex version $H(x, p)$ is

$$
H \equiv H_{1}+i H_{2}=-\frac{i}{2}\left(p^{2}-x^{2}\right) .
$$

From the examples above one can make the following observations:

(a) From Examples (i) - (iii) it can be seen that the role of couplings in $H_{1}$ and $H_{2}$ interchanges. For example, $\beta$ and $\gamma$ in (11) become $\gamma$ and $-\beta$ in (14). Similarly, $\alpha$ and $\beta$ in (19) become $-\beta$ and $\alpha$ in (20).

(b) Angular momentum-type terms, namely $\left(p_{1} x_{2}-p_{2} x_{1}\right)$ in $H_{1}$ (cf. Examples (ii) and (iv) ) transform into the form $\frac{1}{2}\left(p_{1}^{2}+p_{2}^{2}-x_{1}^{2}-x_{2}^{2}\right)$ in $H_{2}$ or vice versa (cf. Example (i)).

(c) It may be emphasized that the $\mathcal{P} \mathcal{T}$-symmetry is not a prerequisite as far as the constructions above are concerned. In fact, none of $H_{1}$ and $H_{2}$ in the examples above (except $H_{2}$ of Example (iv)) is $\mathcal{P} \mathcal{T}$-symmetric, i.e. they are not invariant under the transformation $\left(x_{1}, x_{2}, p_{1}, p_{2}\right) \rightarrow\left(-x_{1},-x_{2}, p_{1}, p_{2}\right)$, $i \rightarrow-i$.

It may be mentioned that we could carry out the constructions above using the prescription of the Bäcklund transformation in the form of the Cauchy-Riemann conditions, which basically are linear first-order partial differential equations. This was possible for a very special class of 'Hamiltonians' $H_{1}\left(x_{1}, x_{2}, p_{1}, p_{2}\right)$. Perhaps the use of other nontrivial forms of the Bäcklund transformation will provide a method to construct invariants for more general cases. 
On the basis of the above analysis of the cases (cf. Table 1) and constructions (cf. Examples (i) - (iv) ) and also of our earlier studies [10] it may be remarked that $\mathcal{P} \mathcal{T}$-symmetry is only a restricted way of complexifying

the one-dimensional Hamiltonian $H(x, p)$. It is rather a special case of the present formulation carried out using the transformation (1).

To summarize, we mention that using the transformation (1) we have demonstrated the viability of the analyticity property of the one-dimensional complex Hamiltonian $H(x, p)$ with regard to the construction of the second integral of motion for a certain class of two-dimensional Hamiltonian systems which can be identified with $H_{1}\left(x_{1}, x_{2}, p_{1}, p_{2}\right)$. This ensures the integrability of this latter class, at least for autonomous systems. No doubt, most of these constructions (cf. Table 1 and Examples (i) - (iv)) turn out to be non-conventional in the sense of physics but still some of them could be of mathematical interest in different branches of theoretical science including biophysics.

Acknowledgements: One of us (RSK) would like to thank Professor H. J. W. Müller-Kirsten for the encouragement and the A. v. Humboldt Stiftung, Bonn, for the financial assistance during the course of this work. RSK also acknowledges the award of Research Scientist Scheme by the UGC, New Delhi.

\section{References}

[1] See, for example, T. J. Hollowwood, Nuclear Phys. B 38 (1992) 523; N. Hatano and D. R. Nelson, Phys. Rev. Lett. 77 (1996) 570; N. Hatano and D. R. Nelson, Phys. Rev. B 56 (1997) 8651.

[2] N. Moiseyev, Phys. Rep. 302 (1998) 211.

[3] A. L. Xavier, Jr. and M. A. M. de Aguiar, Ann. Phys. (N.Y.) 252 (1996) 458.

[4] A. L. Xavier, Jr. and M. A. M. de Aguiar, Phys. Rev. Lett. 79 (1997) 3323; M. A. M. de Aguiar, M. Baranger, F. Keck, H. J. Korsch, and B. Schellhaaß, J. Phys. A (2000), submitted.

[5] C. M. Bender and S. Boettcher, Phys. Rev. Lett. 80 (1998) 5243. 
[6] C. M. Bender and S. Boettcher ans P. N. Meisinger, J. Math. Phys. 40 (1999) 2201.

[7] F. M. Fernandez, R. Guardiola, J. Ros, and M. Znojil, J. Phys. A 31 (1998) 10105.

[8] A. Khare and P. Mandal, Phys. Lett. A 272 (2000) 53.

[9] See, for example, R. K. Colgrave, P. Coxson, and M. A. Mannan, Phys. Lett. A 131 (1988) 407.

[10] R. S. Kaushal and Shweta Singh, Construction of Complex Invariants for Classical Dynamical Systems, to be published.

[11] M. S. Child, Semiclassical mechanics with molecular applications, Oxford University Press, Oxford, 1991.

[12] H. J. Korsch, in Resonances, Springer Lecture Notes in Physics (Vol. 325), edited by E. Brändas and N. Elander, page 253. Springer, 1989.

[13] M. Ozorio de Almeida, Hamiltonian Systems - Chaos and Quantization, Cambridge University Press, Cambridge, 1988.

[14] V. S. Vladimirov, Methods of the Theory of Functions of Many Complex Variables, The MIT Press, Cambridge, Mass., 1966.

[15] J. Hietarinta, Phys. Rep. 147 (1987) 87; R. S. Kaushal, Classical and Quantum Mechanics of Noncentral Potentials: A survvey of TwoDimensional Systems, (jointly published by Narosa Pub. House, New Delhi, and Springer Verlag, Heidelberg, 1998), Chap.2.

[16] P. G. Drazin and R. S. Johnson, Solitons: An Introduction, Cambridge Univ. Press, Cambridge, 1990.

[17] G. 't Hooft, in Basics and Highlights of Fundamental Physics, Erice, 1999, preprint no. hep-th/0003005 (May 2000); see also Class. Quant. Grav. 16 (1999) 3263.

[18] M. Blasone, P. Jizba, and G. Vitiello, Dissipation and Quantization, preprint no. hep-th/0007138 (July 2000). 
[19] M. Blasone, P. Jizba, and G. Vitiello, Quantum theory and dissipative deterministic systems, in Proc. XIII Int. Cong. on Math. Phys., Imperial College, London, July 2000, abstract no. QMS-P5-44.

Table 1: Hamiltonian structures $H=H_{1}+i H_{2}$ which conform to Eq. (5).

Hamiltonian $H$

1. $\frac{1}{2} p^{2}+\frac{1}{2} \omega^{2} x^{2}$

2. $p^{2}+x^{2}+i \gamma x$

3. $p^{2}+\delta_{1}(i x)+\delta_{2}(i x)^{2}$

$$
+\delta_{3}(i x)^{3}
$$

4. $\frac{1}{2} p^{2}+\frac{1}{2} \omega^{2}(t) x^{2}$

5. $\frac{1}{2} p^{2}+\frac{1}{2} a x^{2}+\frac{1}{4} b x^{4}$

6. $p^{2}+i x^{3}+i x$

7. $p^{2}-(\zeta \cosh 2 x-i M)^{2}$

8. $p^{2}+i x^{3}+x$
Real Part $H_{1}$

$$
\begin{aligned}
& \frac{1}{2}\left(p_{1}^{2}-x_{2}^{2}+\omega^{2} x_{1}^{2}-\omega^{2} p_{2}^{2}\right) \\
& p_{1}^{2}-x_{2}^{2}+x_{1}^{2}-p_{2}^{2}-\gamma p_{2} \\
& p_{1}^{2}-x_{2}^{2}-\delta_{1} p_{2}-\delta_{2}\left(x_{1}^{2}-p_{2}^{2}\right) \\
& \quad-\delta_{3} p_{2}^{3}+3 \delta_{3} x_{1}^{2} p_{2}
\end{aligned}
$$$$
\frac{1}{2}\left(p_{1}^{2}-x_{2}^{2}+\omega^{2}(t) x_{1}^{2}-\omega^{2}(t) p_{2}^{2}\right)
$$$$
\frac{1}{2}\left(p_{1}^{2}-x_{2}^{2}\right)+\frac{1}{2} a\left(x_{1}^{2}-p_{2}^{2}\right)
$$$$
+\frac{1}{4} b\left(x_{1}^{4}+p_{2}^{4}-6 x_{1}^{2} p_{2}^{2}\right)
$$$$
p_{1}^{2}-x_{2}^{2}+p_{2}^{3}-3 x_{1}^{2} p_{2}-p_{2}
$$$$
p_{1}^{2}-x_{2}^{2}-\frac{\zeta^{2}}{2}\left(\cos 4 p_{2} \cosh 4 x_{1}+1\right)
$$$$
+M^{2}-2 M \zeta \sin 2 p_{2} \sinh 2 x_{1}
$$$$
p_{1}^{2}-x_{2}^{2}+p_{2}^{3}-3 x_{1}^{2} p_{2}+x_{1}
$$

Imaginary Part $\mathrm{H}_{2}$

$$
p_{1} x_{2}+\omega^{2} x_{1} p_{2}
$$$$
2 p_{1} x_{2}+2 x_{1} p_{2}+\gamma x_{1}
$$$$
2 p_{1} x_{2}+\delta_{1} x_{1}-2 \delta_{2} x_{1} p_{2}
$$$$
-\delta_{3} x_{1}^{3}+3 \delta_{3} x_{1} p_{2}^{2}
$$

$p_{1} x_{2}+\omega^{2}(t) x_{1} p_{2}$

$p_{1} x_{2}+a x_{1} p_{2}+b x_{1}^{3} p_{2}$

$$
-b x_{1} p_{2}^{3}
$$

$2 p_{1} x_{2}+x_{1}^{3}-3 x_{1} p_{2}^{2}+x_{1}$

$2 p_{1} x_{2}-\frac{\zeta^{2}}{2} \sin 4 p_{2} \sinh 4 x_{1}$

$$
+2 M \zeta \cos 2 p_{2} \cosh 2 x_{1} \text { [8] }
$$

$2 p_{1} x_{2}+x_{1}^{3}-3 x_{1} p_{2}^{2}+p_{2}$ 